\title{
USP2I promotes cell proliferation and metastasis through suppressing EZH2 ubiquitination in bladder carcinoma
}

This article was published in the following Dove Press journal:

OncoTargets and Therapy

7 February 2017

Number of times this article has been viewed

\author{
Yong Chen' \\ Bo Zhou ${ }^{2}$ \\ Daihui Chen' \\ 'Department of Urology, The First \\ Affiliated Hospital of Chongqing \\ Medical University, ${ }^{2}$ Department \\ of Urology, Daping Hospital, Third \\ Military Medical University, Chongqing, \\ People's Republic of China
}

\begin{abstract}
Bladder cancer (BC) is the second most common malignant tumor of the urinary tract in the world. In this study, we found that ubiquitin-specific protease (USP21) was upregulated in BC and the ectopic expression of USP21 was closely associated with tumor size and metastasis. Moreover, patients with higher levels of USP21 had poorer survival rate. Multiple function analysis such as CCK-8, colony formation, wound healing, and transwell analysis indicated that USP21 regulated cell proliferation and metastasis in bladder carcinoma cell lines. We also found that USP21 could facilitate epithelial-mesenchymal transition. As EZH2 has been reported to promote cell metastasis in BC, our work identified that USP21 deubiquitinated EZH2 and stabilized it. Our data demonstrated that USP21 might play a crucial role in regulating $\mathrm{BC}$ progression and could provide a potential therapeutic strategy for $\mathrm{BC}$.
\end{abstract}

Keywords: USP21, proliferation, metastasis, EZH2, EMT

\section{Introduction}

Bladder cancer $(\mathrm{BC})$ has become the second most common malignant tumor of the urinary tract in the world. ${ }^{1,2}$ The urothelial carcinoma of the bladder is the most common type and accounts for $~ 95 \%$ of bladder carcinoma. ${ }^{3}$ Approximately $47 \%$ of transitional cell carcinoma recur as a nonlethal disease initially and $\sim 9 \%$ will ultimately deteriorate to a muscle-invasive bladder carcinoma, ${ }^{1,2}$ which commonly occurs as metastasis and results in a high rate of death. ${ }^{3}$ In patients with invasive and metastatic bladder carcinoma, the traditional therapy slightly improves the 5 -year survival rate. ${ }^{4}$ In the recent decade, although many targeted therapies have excelled in several cancers, such as gefitinib in lung carcinoma and sunitinib in kidney carcinoma, there is still no evidence of the efficiency of targeted therapeutic reagent for bladder carcinoma. ${ }^{1,2,5}$ Hence, finding and developing a more efficient therapeutic target is urgently needed. Meanwhile, it is crucial to investigate the molecular mechanism of $\mathrm{BC}$ development in detail and it is beneficial for finding a potential molecular target for bladder carcinoma therapy.

Protein ubiquitination involves multiple cellular processes, such as protein degradation, transcriptional activation or inhibition, and immune signal transduction pathways. ${ }^{6-8}$ The deubiquitinase (DUB) family has been found in at least 79 members in humans. There are five subfamilies of DUB: ovarian tumor, ubiquitin C-terminal hydrolases, ubiquitin-specific proteases (USPs), Josephin domain, and JAB1/MPN/ MOV34 proteases (JAMM) family. ${ }^{9,10}$ For example, USP7 and USP10 were found to regulate $\mathrm{p} 53$ localization and function. ${ }^{11,12}$ USP15, USP21, and USP31 have been found to play key roles in the regulation of the NF- $\mathrm{KB}$ pathway. ${ }^{13-18}$
Correspondence: Yong Chen Department of Urology, The First Affiliated Hospital of Chongqing Medical University, No I Friendship Road, Yuzhong District, Chongqing 4000I6, People's Republic of China

Tel +8623688I 1360

Email tiantiankaixin200@yeah.net 
USP21 belongs to the USPs family with a C-terminal catalytic DUB domain. ${ }^{19}$ USP2 1 has been regarded as an USP, which catalyzes the hydrolysis of $\mathrm{ubH} 2 \mathrm{~A}$ and activates tran-

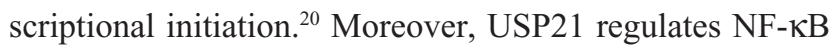
signaling pathway or Th2-specific transcriptional factor GATA3 to modulate immune defense. ${ }^{18,21}$ Recently, one report indicated that USP21 promotes cell proliferation and invasion ability in human renal cell carcinoma. ${ }^{22}$ However, the role of USP21 in bladder carcinoma is still unknown.

In our study, we suggested that USP21 was an oncogene in bladder carcinoma because it had an obviously high expression in BC tissue samples and cell lines; moreover, high expression of USP21 was closely associated with tumor size, metastasis, and poor prognosis. In addition, several functional experiments indicated that USP21 not only promoted cell proliferation but also facilitated metastasis through regulation epithelial-mesenchymal transition (EMT) process. Furthermore, we identified that USP21 directly regulated the protein level of EZH2 through its DUB activity. These findings demonstrated that USP2 1 could enhance the progression of bladder carcinoma and provide a novel potential targets for bladder carcinoma therapy.

\section{Methods and materials}

\section{Cell culture and transfection}

Human-immortalized bladder urothelial cell line (SV-HUC-1) and human BC cell lines (T24 and 5637) were purchased from Shanghai Cell Bank (People's Republic of China) and cultured in Roswell Park Memorial Institute 1640 medium (HyClone, Logan, UT, USA) supplemented with 1\% penicillin-streptomycin (HyClone) and 10\% fetal bovine serum (HyClone) at $37^{\circ} \mathrm{C}$ with $5 \% \mathrm{CO}_{2}$.

Lipofectamine 2000 (Invitrogen, Carlsbad, CA, USA) was used to transfect cells according to the manufacturer's protocol. In brief, DNA plasmids or USP21 siRNA were mixed with Opti-MEM medium and lipofectamine 2000 reagents, gently vortexed and stranded for $10 \mathrm{~min}$ at room temperature. After adding it to the cells, the medium was replaced $6 \mathrm{~h}$ later. The transfection efficiency was determined after $48 \mathrm{~h}$.

\section{Bladder tissue samples}

The 62 adjacent normal bladder tissues and BC tissues were obtained from The First Affiliated Hospital of Chongqing Medical University during 2015-2016. All patients were aware that their tissue sample would be used for research prior to the study and had provided written informed consent for the samples to be used. Our research was approved by the Institutional Research Ethics Committee of The First Affiliated
Hospital of Chongqing Medical University (FAHCM201503). All the tissue samples were collected and frozen in liquid nitrogen and stored at $-80^{\circ} \mathrm{C}$ until processing.

\section{Quantitative real time polymerase chain reaction ( $q R T-P C R$ )}

RNeasy mini kit (Qiagen, Hilden, Germany) was used to extract total RNA from $40 \mathrm{mg}$ tumor or normal tissue samples and cells and next cDNA was synthesized using PrimeScript RT reagent kit (Takara, Tokyo, Japan). For real-time qPCR, we used the 7500 Real-Time PCR System using SYBR ${ }^{\circledR}$ Green (Thermo Fisher Scientific, Waltham, MA, USA). The relative expression of mRNA was normalized against $\beta$-actin. All experiments were repeated at least three times.

\section{Western blotting}

After collecting and lysating cells in radio immunoprecipitation assay lysis buffer (BLKW, Fanke Biological Technology Co., Ltd, Shanghai, People's Republic of China), we next measured protein concentrations with bicinchoninic acid assay kit (Pierce, Appleton, WI, USA). Equal amounts of protein were separated in $8 \%$ sodium dodecyl sulfate polyacrylamide gel electrophoresis (SDS-PAGE) and transferred proteins to polyvinylidene difluoride membranes (Millipore, Bedford, MA, USA). After blocking the membranes with 5\% nonfat dry milk in Tris-buffered saline, Tween 20 (TBST) buffer, membranes were incubated with indicated primary antibodies at $4^{\circ} \mathrm{C}$ overnight. We washed the membranes with TBST buffer three times and incubated membranes with the horseradish peroxidase-labeled secondary antibodies at room temperature for $1 \mathrm{~h}$. After washing the membranes with TBST buffer three times, the immunobands were visualized using the ECL reagents (ECL-Amersham, Piscataway, NJ, USA).

\section{CCK-8 assays}

The CCK-8 assay was used to determine the cell proliferation ability. After cells were transfected with vector, USP21, scramble siRNA (SCR), siUSP21 for $48 \mathrm{~h}$, collected, and counted, $4 \times 10^{3}$ cells were placed in 96 -well plates with $100 \mu \mathrm{L}$ complete medium. Every $24 \mathrm{~h}, 10 \mu \mathrm{L}$ CCK-8 were added in each well and then incubated at $37^{\circ} \mathrm{C}$ for $30 \mathrm{~min}$. The absorbance was measured at $450 \mathrm{~nm}$ to assess the number of viable cells. All experiments were repeated at least three times.

\section{Colony formation assay}

Colony formation assay was used to determine the cell proliferation ability. After cells were transfected with vector, USP21, SCR, siUSP21 for $48 \mathrm{~h}$, collected and counted, 
$3 \times 10^{3}$ cells were placed in six-well plates and incubated for 14 days with complete medium. The colonies were fixed with $10 \%$ formaldehyde and stained with $0.5 \%$ crystal violet. The colonies were counted under microscope. All experiments were repeated at least three times.

\section{Transwell assay}

Transwell assay was performed to detect the cell invasiveness ability. We used transwell chambers with $8 \mu \mathrm{m}$ pores (Corning Costar, Corning, NY, USA) and Matrigel (BD, San Diego, CA, USA), and coated matrigel on chambers. After cells were transfected with vector, USP21, SCR, siUSP21 for $48 \mathrm{~h}$, collected and counted, $2 \times 10^{3}$ cells were placed on top chambers with serum-free medium, whereas the lower chamber completes with the medium. After incubating cells at $37^{\circ} \mathrm{C}$ with $5 \% \mathrm{CO}_{2}$ for $24 \mathrm{~h}$, cotton swabs were used to remove the cells remained in the upper side, fixed and stained with $0.5 \%$ crystal violet. Cells were counted under microscope. All experiments were repeated at least three times.

\section{Immunopurification and mass spectrometry (MS)}

We overexpressed FLAG-USP21 in 5637 cells after 48 h of transfection, lysated whole cells, added $70 \mu \mathrm{L}$ of $50 \%$ FLAG column, and incubated for $4 \mathrm{~h}$ at $4^{\circ} \mathrm{C}$. After washing the column with phosphate-buffered saline (PBS) for three times, subsequently FLAG peptides (Sigma, New York, USA) were used to elute protein complex. The eluted solution was resolved on SDS-PAGE gel, stained gel with silver-stained kit (Pierce), and the special band were subjected to liquid chromatography-MS/MS sequencing.

\section{Co-immunoprecipitation (co-IP)}

For co-IP assays, we lysated whole cells by NETN lysis buffer (0.5 mM ethylenediaminetetraacetic acid [EDTA], $20 \mathrm{mM}$ Tris- $\mathrm{Cl}$ [pH 8.0], $100 \mathrm{mM} \mathrm{NaCl}, 1 \times$ cocktail protease inhibitor, $0.5 \%$ Nonidet P-40 [NP-40]) and next incubated with $2 \mu \mathrm{g}$ anti-USP2 1 or anti-EZH2 antibody and $-35 \mu \mathrm{L}$ protein A-agarose beads at $4^{\circ} \mathrm{C}$ overnight. We washed the beads with NETN lysis buffer three times and eluted immunocomplexes via boiling in SDS sample buffer at least for $10 \mathrm{~min}$. Finally, Western blotting was performed to identify protein.

\section{GST pull-down analysis}

We utilized $40 \mu \mathrm{L}$ of $50 \%$ glutathione-Sepharose $4 \mathrm{~B}$ beads (Solarbio, Beijing, People's Republic of China) to immobilize GST fusion proteins in $500 \mu \mathrm{L}$ binding buffer $(5 \%$ glycerol, $100 \mathrm{mM} \mathrm{KCl,} 10 \mathrm{mM}$ Hepes, 5 mM EDTA, $3 \mathrm{mM}$ $\mathrm{MgCl}_{2}, 0.5 \% \mathrm{CA} 630$, and $3 \mathrm{mM} \mathrm{MgCl}_{2}$ ). We incubated
GST fusion proteins and beads for $2 \mathrm{~h}$ at $4^{\circ} \mathrm{C}$ with rotation, washed beads with binding buffer for three times, and next resuspended and added $5 \mu \mathrm{L}$ transcribed/translated EZH2, $4 \%$ bovine serum albumin and again incubated it for at least $90 \mathrm{~min}$ at $4^{\circ} \mathrm{C}$ with rotation. We washed beads with ice-cold PBS for three times and boiled the beads with $35 \mu \mathrm{L}$ of $2 \times$ SDS loading buffer and resolved on 10\% SDS-PAGE.

\section{Statistical analysis}

The GraphPad Prism 5 statistical software (GraphPad Software, La Jolla, CA, USA) was used to evaluate all the statistical analyses. The correlation between USP21 expression and patients' clinical pathological characteristics was evaluated by chi-square test. The two-tailed Student's $t$-test was used to assess the differences between two groups. The $* P<0.05$ was considered statistically significant. All experiments were repeated at least three times, and the values were shown as mean \pm standard deviation.

\section{Results}

\section{USP2I is highly expressed in BC tissues and cell lines and predicts poor prognosis of $B C$}

To investigate the function of USP21 in BC, we first performed qRT-PCR to assess the expression level of USP21 in $\mathrm{BC}$ tissues and adjacent normal tissues. To our surprise, we found that USP21 was elevated in BC tissues compared with the adjacent normal tissues (Figure 1A). In addition, we further decipher the relationship of the expression level of USP21 with the pathological characteristics of BC. As shown in Table 1, high expression of USP21 was more closely correlated with high-grade $\mathrm{BC}$ than in low-grade tumors. Moreover, USP21 was found to be evaluated in high tumor stage, suggesting a potential relationship of tumor progression with the expression of USP21. Tumor size and lymphatic invasion also had association with high expression of USP 21. This finding suggested that USP21 played an important role in tumor growth and metastasis in BC. According to the KM plot, we draw the survival curve. The survival curve revealed that the patient who had high expression of USP21 had a shorted survival time than the patient who had low expression of USP21 (Figure 1B). Together, our work revealed that the elevated expression of USP21 was closely correlated with the tumor aggressiveness, eventually resulted in poor prognoses. Subsequently, we determined the expression of USP21 in two BC cell lines (5637 and T24) and normal urothelial cell line (SV-HUC-1) as control. As shown in Figure 1C and D, both the mRNA level and the protein level of USP21 were highly expressed in BC cell lines, compared with SV-HUC-1. 
A
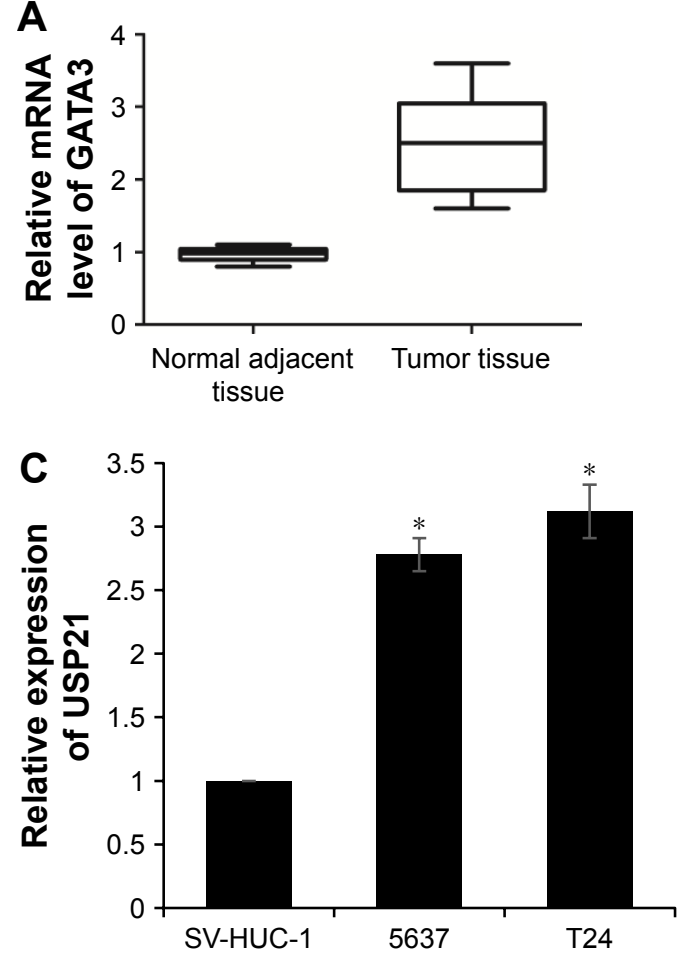

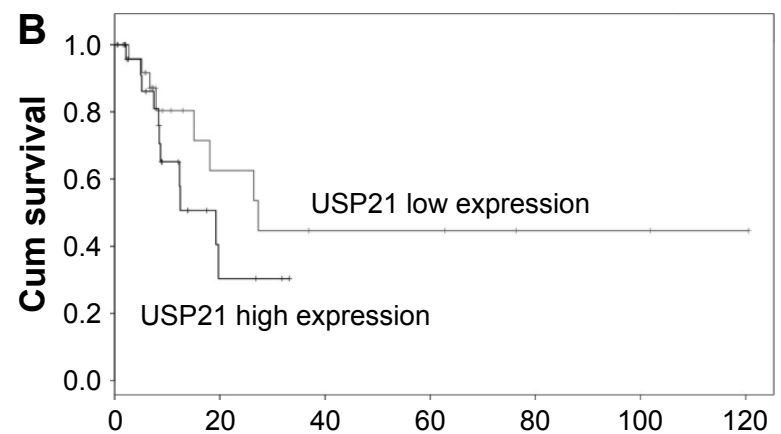

D

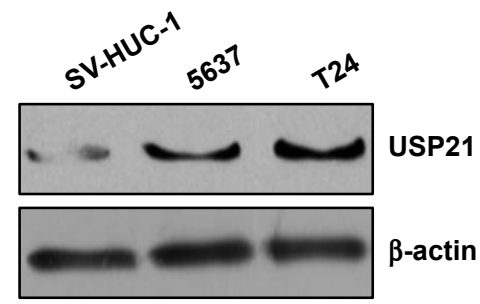

Figure I USP2I is highly expressed in BC tissues and cell lines, and it predicted poor prognosis of BC.

Notes: (A) The mRNA level of USP2I in adjacent normal tissue and tumor tissue was detected by qRT-PCR. These results revealed that USP2I has an obviously high expression in BC tissues. (B) According to Kaplan-Meier method of analysis, the correlation of USP2I with survival curves. (C) The mRNA level of USP2I in normal urothelial cell line (SV-HUC-I) and two BC cell lines (5637 and T24) was analyzed by qRT-PCR. *Compared with SV-HUC-I, P<0.05. (D) The protein level of USP2I in normal urothelial cell line (SV-HUC-I) and two BC cell lines (5637 and T24) was analyzed by Western blotting.

Abbreviations: BC, bladder cancer; HUC, human urothelial cell; USP, ubiquitin-specific protease.

Table I Clinicopathologic variables in 66 bladder cancer patients

\begin{tabular}{|c|c|c|c|c|}
\hline \multirow[t]{2}{*}{ Variables } & \multirow[t]{2}{*}{$N=66$} & \multicolumn{2}{|c|}{ USP2I protein expression } & \multirow[t]{2}{*}{$P$-value } \\
\hline & & Low $(n=23)$ & High $(n=43)$ & \\
\hline Age (years) & & & & 0.423 \\
\hline$<60$ & 30 & 12 & 18 & \\
\hline$\geq 60$ & 36 & 11 & 25 & \\
\hline Drinking & & & & 0.073 \\
\hline Yes & 36 & 16 & 20 & \\
\hline No & 30 & 7 & 23 & \\
\hline Tumor size $(\mathrm{cm})$ & & & & 0.001 \\
\hline Small $(\leq 1.5)$ & 33 & 18 & 15 & \\
\hline Large $(\geq \mid .5)$ & 33 & 5 & 28 & \\
\hline Metastasis & & & & 0.006 \\
\hline Yes & 38 & 8 & 30 & \\
\hline No & 28 & 15 & 13 & \\
\hline Histologic grades & & & & 0.037 \\
\hline$|-| I$ & 26 & 13 & 13 & \\
\hline III-IV & 40 & 10 & 30 & \\
\hline $\mathrm{p} T$ & & & & 0.007 \\
\hline $\mathrm{I}-2$ & 31 & 16 & 15 & \\
\hline $3-4$ & 35 & 7 & 28 & \\
\hline $\mathrm{pN}$ & & & & 0.032 \\
\hline 0 & 34 & 16 & 18 & \\
\hline I & 32 & 7 & 25 & \\
\hline Differentiation & & & & 0.351 \\
\hline Well/moderate & 31 & 9 & 22 & \\
\hline Poor & 35 & 14 & 21 & \\
\hline
\end{tabular}

Abbreviation: USP, ubiquitin-specific protease.

\section{Overexpression of USP2I promotes} proliferation of BC cells

Our previous work indicated that high expression of USP21 was coordinated with tumor size, hence, we next investigated the involvement of USP21 in tumor progression. BC cell lines 5637 and T24 were transfected with vector, USP21 plasmid, SCR, or USP21 siRNA, and the expression of USP21 were detected. As shown in Figure 2A and B, the USP21 siRNA\#1 was more efficient than USP21 siRNA\#2, thus the subsequent experiments used USP21 siRNA\#1. After transfection, we performed colony formation analysis and CCK-8 analysis to evaluate the functions of USP21 on cell proliferation. As shown in Figure $2 \mathrm{C}$ and $\mathrm{D}$, ectopic expression of USP21 obviously increased the proliferation rates of 5637 and T24 cells, whereas the inhibition of USP21 expression significantly inhibited cell proliferation. Together, our findings demonstrated that USP21 promoted cell proliferation in $\mathrm{BC}$.

\section{USP2I promotes migration and invasion ability of BC cells and facilitates EMT}

Because lymphatic invasion also had association with high expression of USP21, we next performed wound healing 

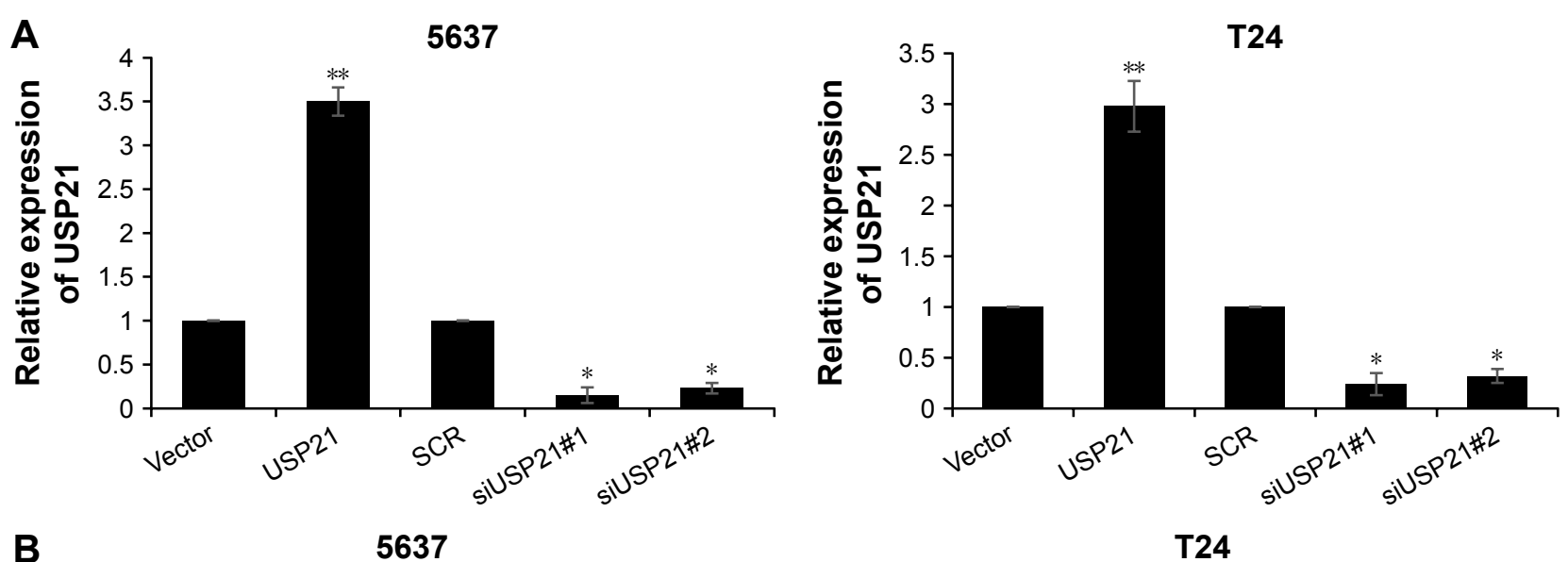

B

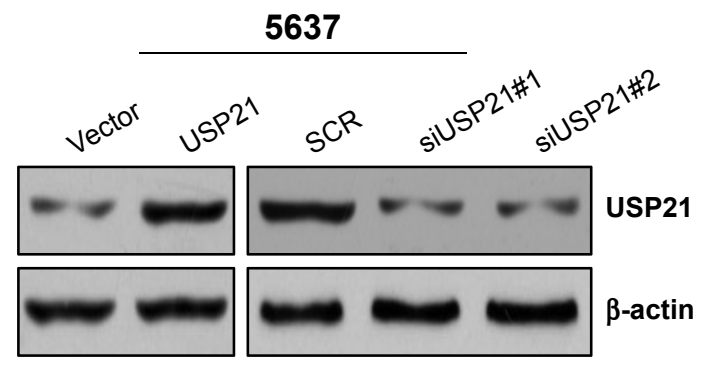

C
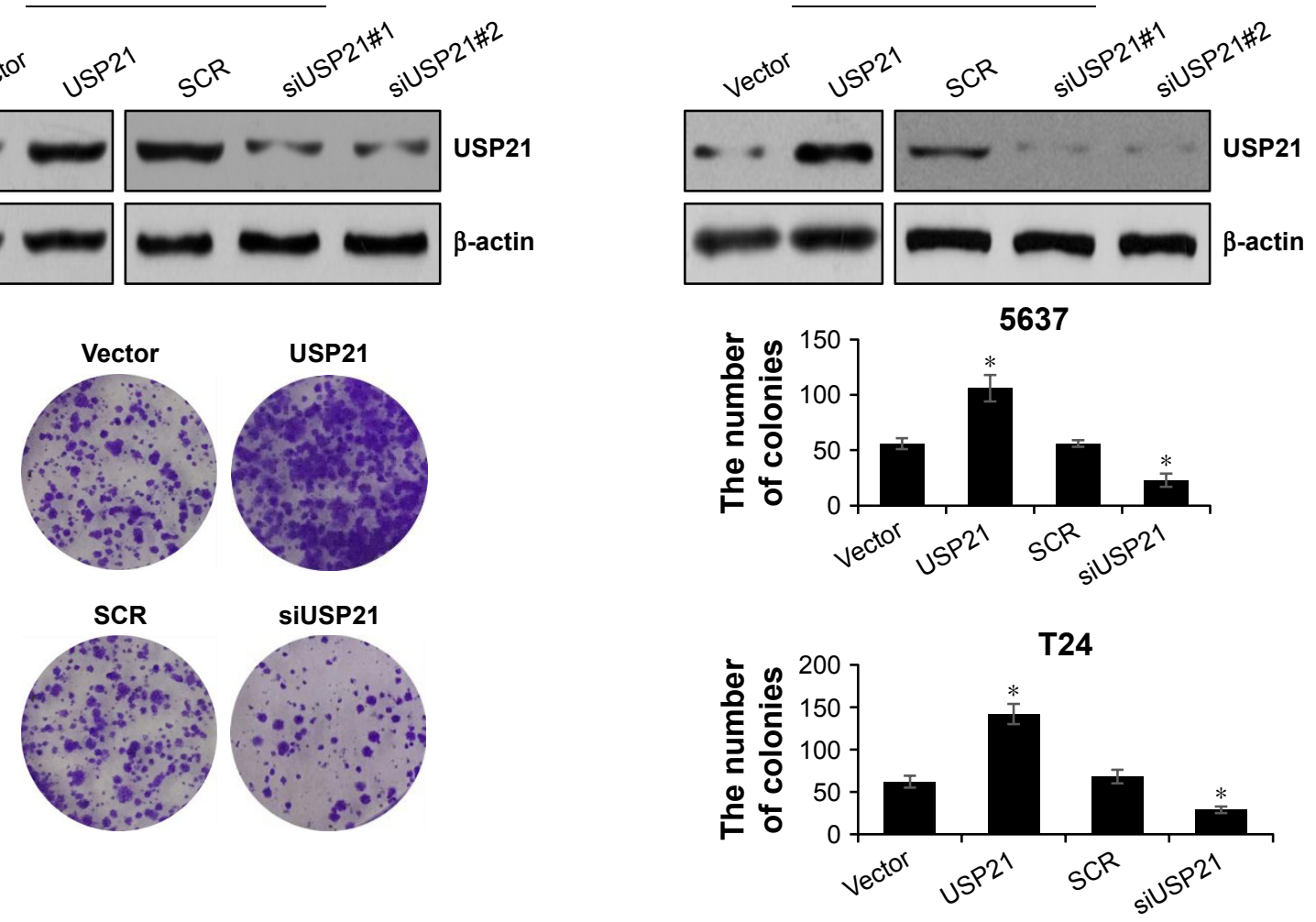

D

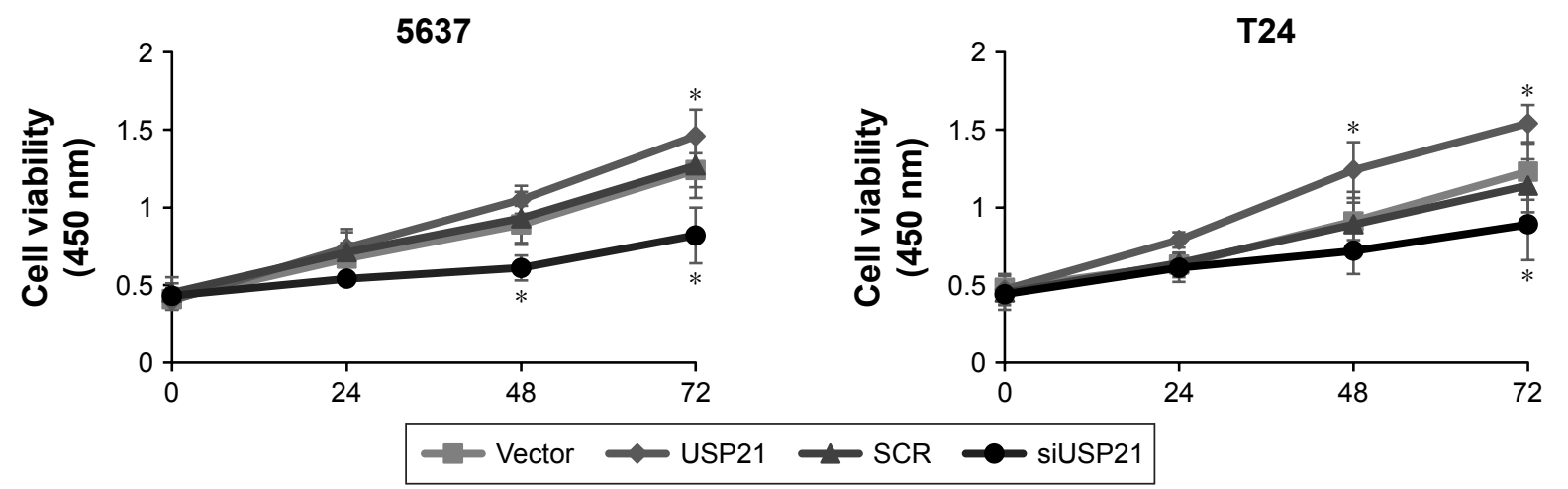

Figure 2 Overexpression of USP2I promotes proliferation of bladder cancer cells.

Notes: (A) After transfected vector or USP2I or SCR or USP2I siRNA in 5637 and T24 cells according to the manufacture's protocol, after $48 \mathrm{~h}$ of transfection, we extracted total RNA and performed qRT-PCR to detect mRNA level of USP2I. (B) After transfected vector or USP2I or SCR or USP2I siRNA in 5637 and T24 cells according to the manufacture's protocol, after $48 \mathrm{~h}$ of transfection, we collected and lysate whole cell, and performed Western blotting to detect protein level of USP2I. (C) After overexpressed or silenced USP2I in 5637 and T24 cells, after $48 \mathrm{~h}$ of transfection, we placed $3 \times 10^{3}$ cells in six-well plates, incubated for 14 days, and the cell were stained with $0.1 \%$ crystal violet and counted. (D) After overexpressed or silenced USP2I in 5637 and T24 cells, we evaluated cell proliferation by CCK-8 assay on the indicated day. *Compared with vector, $P<0.05$; **Compared with vector, $P<0.01$.

Abbreviations: SCR, scramble siRNA; USP, ubiquitin-specific protease. 
analysis and transwell assay to determine the effect of USP21 in cell migration and invasion. As shown in Figure 3A, the cells with ectopic expression of USP 21 migrated to $\sim 100 \%$ of the wounded area, whereas the control groups migrated $\sim 50 \%$ of the area. However, the cells with the inhibition of USP21 migrated to $\sim 17 \%$ of the wounded area, whereas the control groups migrated $\sim 50 \%$ of the area. The transwell assay revealed that the number of invaded cells was significantly increased in the USP21 overexpression group compared with the control group. The number of invaded cells was significantly increased in the USP21-depleted group compared with the control group (Figure 3B). These data revealed that USP21 not only promoted cell proliferation but also facilitated cell migration and invasion in $\mathrm{BC}$. To further decipher the molecular mechanism of USP21 on BC cell migration and invasion, we aimed to identify whether USP21 regulated EMT. To verify our hypothesis, we assessed several EMT markers (E-cadherin, $\alpha$-catenin, $N$-cadherin, and fibronectin) by qRT-PCR and Western blot. As expected, we found that E-cadherin and $\alpha$-catenin were remarkably decreased and $\mathrm{N}$-cadherin and fibronectin were obviously increased while overexpressing USP21 (Figure 3C). The contrary results were observed while USP21 was depleted (Figure 3D). To investigate whether USP21 regulated EMT through its DUB activity, we added MG132 in USP21-depleted cells. We found that the changes in epithelial and mesenchymal markers were obviously reverse (Figure 3D). These results suggested that USP21 facilitated BC cell metastasis through the promotion of the EMT process.

\section{Identifying USP2I as a EZH2-interacting protein}

To further decipher the cellular functions of USP21, we performed affinity purification to detect USP21-interacting protein through MS. We found that there were 11 matching peptides from EZH2, which suggested that USP21 was associated with EZH2 in vivo. EZH2 was reported to have a high expression in BC and correlated with EMT. ${ }^{23-26}$ Subsequently, we verified the interaction between USP21 and EZH2 through co-IP analysis. We performed co-IP assays in 5637 cell lysates with anti-USP21 antibody and then immunoblotting with antibodies against USP21 and EZH2. The results revealed that EZH2 was efficiently interacted with USP21 (Figure 4A). Reciprocal immunoprecipitation with anti-EZH2 and immunoblotting with EZH2 and USP21 also confirmed that USP21 interacts with EZH2 in vivo (Figure 4B). To further determine whether USP21 was directly interacted with EZH2, GST pull-down assay was performed. We expressed GST-USP21 in bacteria and then incubated with transcribed/translated FLAG-EZH2 (Figure 4C). The result showed that USP21 could interact with EZH2 directly in vitro. Together, our works suggested that USP21 was physically associated with EZH2 in vivo.

\section{USP2I stabilizes EZH2 through its ubiquitinase activity}

As a DUB, USP21 was reported to deubiquitinate Gli1 and RIG-I; ${ }^{27,28}$ therefore, we assumed that USP21 may regulate EZH2 and some protein, which was associated with EMT. We first detected several transcriptional factors, such as snail, slug, and twist, but there were no correlation between USP21 and those transcription factors, whereas the protein level of EZH2 was significantly upregulated while overexpressing USP21 (Figure 5A), but the mRNA level of EZH2 had no change (Figure 5B). The above data indicated that USP21 may regulate EZH2 through its DUB activity. To verify our hypothesis, we ectopically expressed wild-type USP21 (wt-USP21) and mutant USP21 (mut-USP21/C221A), which were catalytically inactive in 5637 cells. As expected, EZH2 only increased in cells with overexpressed wt-USP21; however, EZH2 had no change in cells with overexpressed mutant USP21 (USP21/C221A; Figure 5C). Moreover, to determine whether mut-USP21/C221A had any effect on EZH2, because EZH2 had no interaction with mut-USP21/C221A. Co-IP assay was performed, as shown in Figure 5D, USP21/C221A could interact with $\mathrm{EZH} 2$. In addition, after the addition of MG132 in USP21-depleted 5637 cells, it was succeeded to rescue EZH2 protein from degradation (Figure 5E). Together, our works revealed that USP21 could stabilize EZH2 through its DUB activity.

\section{Discussion}

Accumulating evidence has suggested that USP21 is closely correlated to the development of cancer. ${ }^{18,21,22,29}$ Many studies have reported that USP21 deubiquitinated H2A and several nonhistone, such as Gli1 and RIG-I. ${ }^{27,28}$

In our study, we found that USP21 was highly expressed in $\mathrm{BC}$ and the expression of USP21 was associated with tumor size, metastasis, and poor survival rate. Several functional experiments, including colony formation analysis and CCK-8 analysis, suggested that overexpression of USP21 promoted cell proliferation and inhibition of USP21 suppressed cell proliferation. However, the details of molecular mechanism remain unknown. Cell proliferation is a complex cell process. It is regulated by multiple proteins, such as cyclin-D1, cyclin-E1, p16, p21, etc. ${ }^{30-32} \mathrm{We}$ assumed that 
A
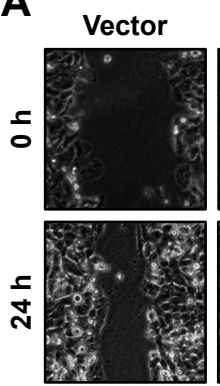

B

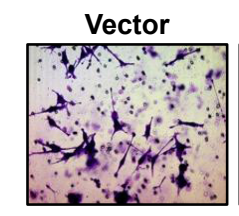

SCR

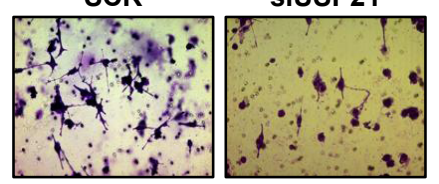

C

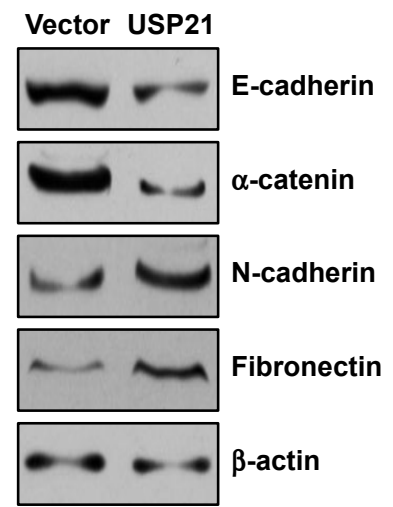

D

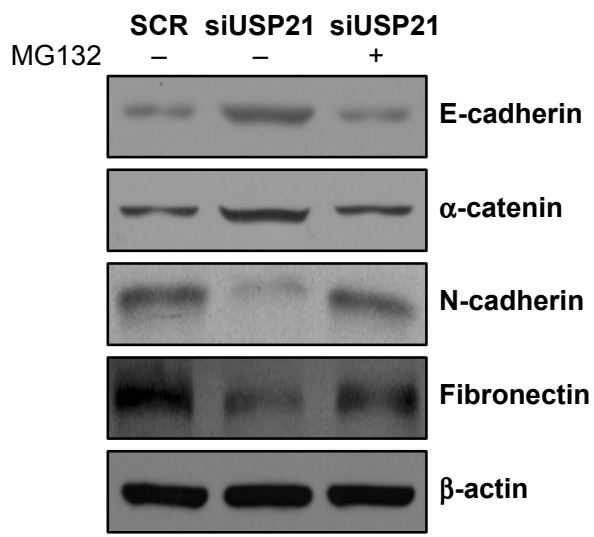

siUSP21
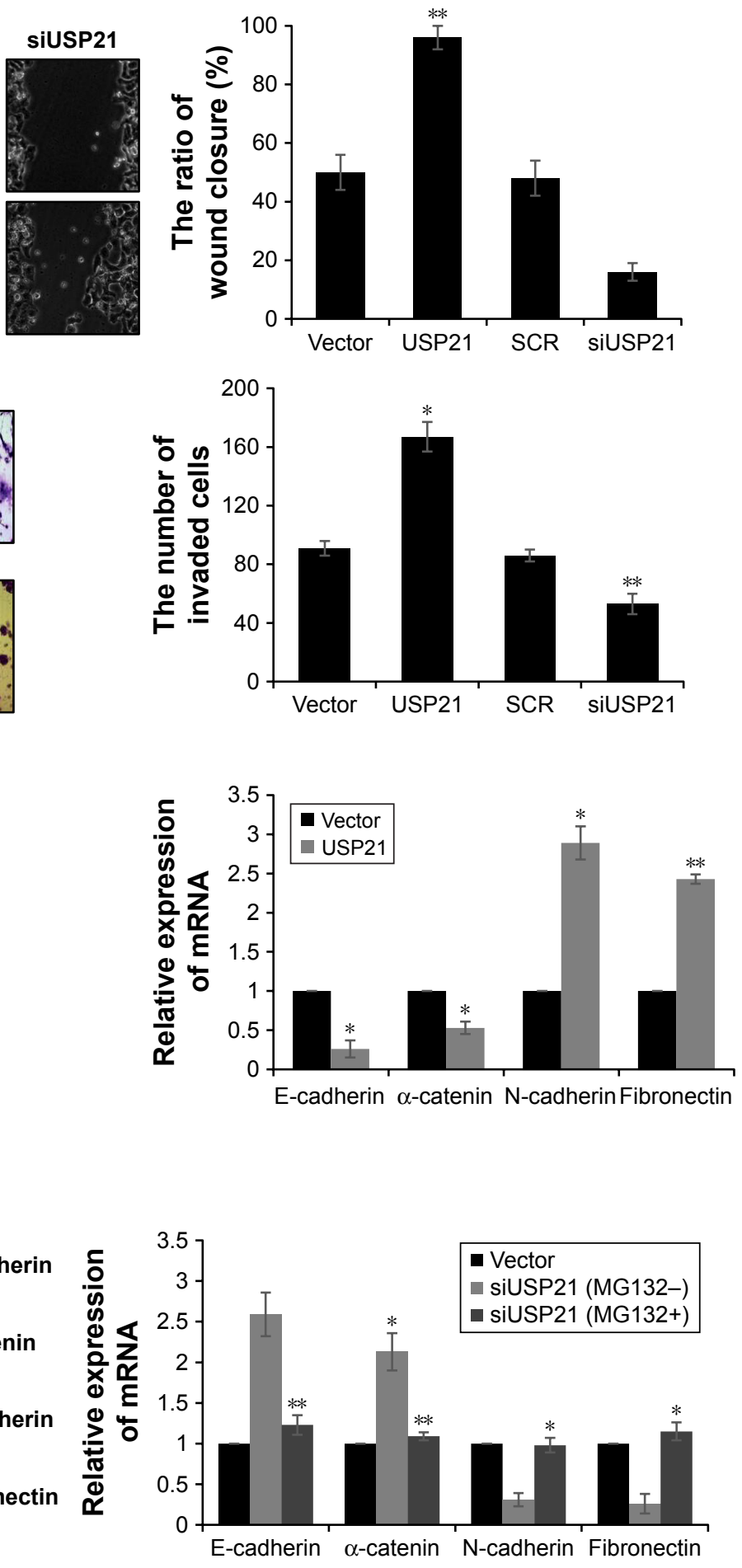

Figure 3 USP2I promotes migration and invasion ability of BC cells and facilitates EMT.

Notes: (A) After overexpressed or silenced USP2I in 5637 cells, $48 \mathrm{~h}$ after transfection, we performed wound healing analysis to assess the migration ability of BC cells and measured the wound closure distance at 0 and $24 \mathrm{~h}$. Overexpression of USP2I promoted the migration ability of BC cells; however, the inhibition of USP2I decreased BC cells migration ability. (B) After overexpressed or silenced USP2I in 5637 cells, $48 \mathrm{~h}$ after transfection, we placed $2 \times 10^{3}$ cells on top chambers with serum-free medium, whereas the lower chamber with complete medium. After incubation, we fixed and stained cells with $0.5 \%$ crystal violet. Overexpression of USP2I promoted the invasion ability of BC cells; however, the inhibition of USP2I decreased BC cells invasion ability. (C) We overexpressed USP2I in 5637 cells and performed Western blotting and qRT-PCR to determine the function of USP2I in EMT process. Overexpression of USP2I decreased the expression of E-cadherin, whereas increased the expression of $\mathrm{N}$-cadherin and fibronectin. (D) We knockdown USP2I in 5637 cells and incubated with or without MGI32, a proteasome-specific inhibitor, and performed Western blotting and qRT-PCR to determine the function of USP2I in EMT process. Although cells were incubated without MGI32, knockdown of USP2I increased the expression of E-cadherin, whereas decreased the expression of N-cadherin and fibronectin. However, while cells were incubated with MGI32, it rescued the effect of USP2I-depleted group. The magnification is $10 \times * P<0.05, * * P<0.01$.

Abbreviations: BC, bladder cancer; EMT, epithelial-mesenchymal transition; SCR, scramble siRNA; USP, ubiquitin-specific protease. 
A

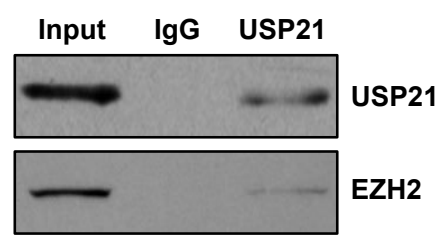

B

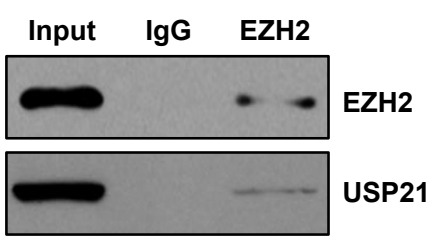

C

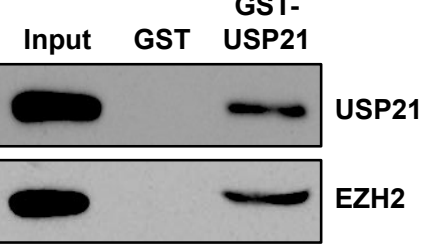

Figure 4 Identifying USP2I as a EZH2-interacting protein.

Notes: (A) Co-IP assay was performed to determine whether USP2I interacted with EZH2 in vivo. 5637 cell lysates were first immunoprecipitated with anti-USP2 I antibody and then immunoblotted using USP2I and EZH2 antibodies. (B) Co-IP assay was performed to determine whether EZH 2 interacted with USP2I in vivo. 5637 cell lysates were first immunoprecipitated with anti-EZH2 antibody and then immunoblotted using EZH2 and USP2I antibodies. (C) GST pull-down analysis was used to determine whether USP2I directly interacted with EZH2 in vitro. We expressed GST-USP2I in bacterially and then incubated with transcribed/translated FLAG-EZH2.

Abbreviations: co-IP, co-immunoprecipitation; USP, ubiquitin-specific protease.

USP21 might regulate some protein, which influenced those cell proliferations. Subsequently, transwell assay and wound healing assay confirmed that USP21 promoted BC cells metastasis. Furthermore, we found that USP21 also facilitated EMT. To further investigate the cellular function of USP21, we performed affinity purification to detect the USP21-interacting protein. To our surprise, EZH2, a component of PRC2 complex, was interacted with USP21.
EZH2 has been reported to have a high expression in several tumors and promote cancer development. Here, co-IP and GST pull-down analysis demonstrated that USP2 1 interacted with EZH2. As a DUB, USP21 deubiquitinated and stabilized several proteins. Next, we detected whether USP21 regulated EZH2 through its DUB activity. The results showed that the ectopic expression of wt-USP21 could stabilize EZH2, but the overexpression of mut-USP21/C221A with
A

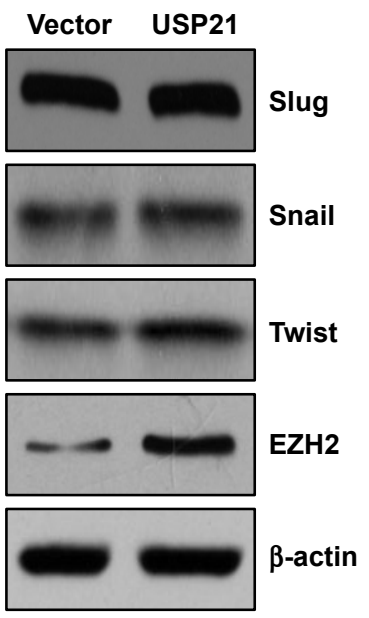

B

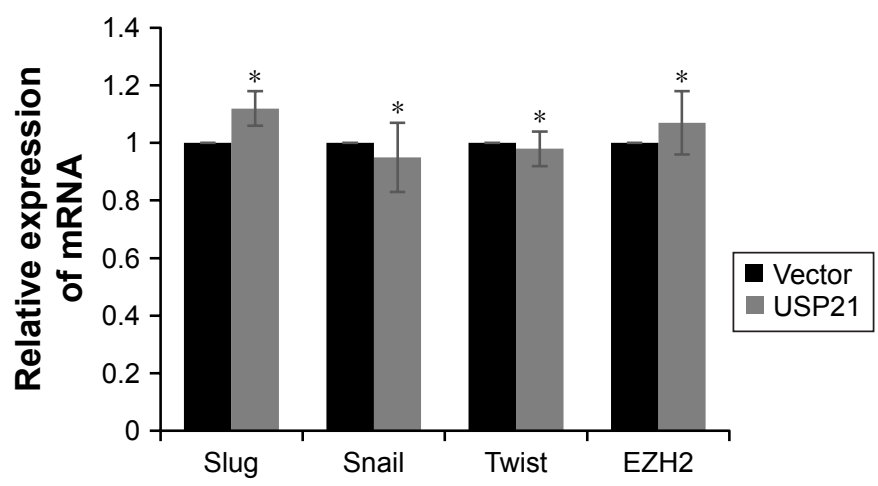

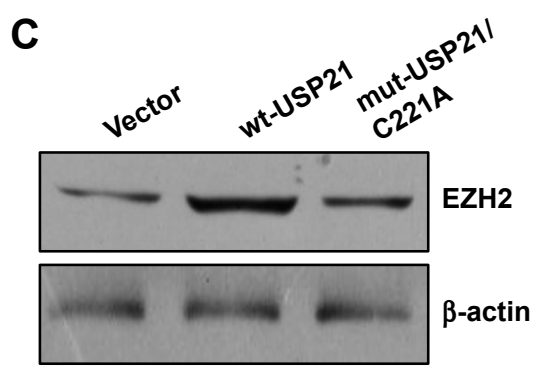

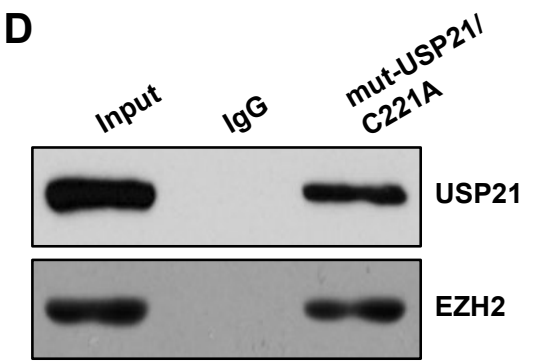

E

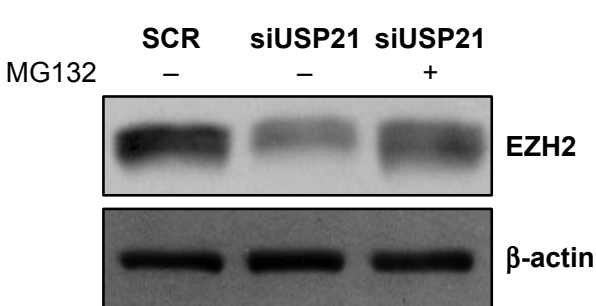

Figure 5 USP2I stabilizes EZH2 through its ubiquitinase activity.

Notes: (A) After overexpressed USP2I in 5637 cells, $48 \mathrm{~h}$ after transfection, Western blotting was used to detect the expression of indicated protein. Overexpression of USP2I had no effect on the expression of snail, slug, and twist, but it increased the expression of EZH2. (B) After overexpressed USP2I in 5637 cells, $48 \mathrm{~h}$ after transfection, qRT-PCR was used to detect the mRNA level of indicated gene. Overexpression of USP2I had no effect on those genes. ${ }^{*}<<0.05$. (C) After overexpressed wide-type USP2I (wt-USP2I) and mutant USP2I (mut-USP2I/C22IA) in 5637 cells, $48 \mathrm{~h}$ after transfection, Western blotting was used to detect the expression of EZH2. Overexpression of wt-USP2I increased the expression of EZH2, whereas the mut-USP2I/C22IA had no effect on the expression of EZH2. (D) After overexpressed mut-USP2I/C22IA in 5637 cells, co-IP analysis was used to assess whether mut-USP2I/C22IA could interact with EZH2. (E) We knockdown the USP2I in 5637 cells and incubated with or without MG 132, and performed Western blotting to detect the protein level of EZH2. While cells were incubated without MGI32, the expression of EZH2 was decreased in USP2I-silenced cells. While cells were incubated with MGI32, the expression of EZH2 had no change in cells in which USP2I was silenced.

Abbreviations: Co-IP, co-immunoprecipitation; USP, ubiquitin-specific protease. 
inactive catalytic had no effect. Moreover, the inhibition of USP2 1 could decrease the expression of EZH2; while adding MG132, the expression of EZH2 was revived. Overall, our study suggests that USP21 stabilized EZH2 through its DUB activity. Meanwhile, USP21-facilitated EMT may also depend on its DUB activity.

In summary, our study demonstrated that USP21 was upregulated in bladder carcinoma and USP21 played an important function in tumor proliferation and metastasis. USP21 should be considered as a potential biomarker for BC.

\section{Disclosure}

The authors report no conflicts of interest in this work.

\section{References}

1. Sylvester RJ. Natural history, recurrence, and progression in superficial bladder cancer. ScientificWorldJournal. 2006;6:2617-2625.

2. Crawford JM. The origins of bladder cancer. Lab Invest. 2008;88(7): 686-693.

3. Kaufman DS, Shipley WU, Feldman AS. Bladder cancer. Lancet. 2009;374(9685):239-249.

4. Bellmunt J, Guix M. New agents for bladder cancer. Ann Oncol. 2010; 21(Supp1 7):vii56-vii58.

5. Resnick MJ, Chang SS. Optimizing outcomes for octogenarians with invasive bladder cancer: one size does not fit all. Urol Oncol. 2013; 31(1):1-4.

6. Hershko A, Ciechanover A. The ubiquitin system. Annu Rev Biochem. 1998;67:425-479.

7. Sun SC. Deubiquitylation and regulation of the immune response. Nat Rev Immunol. 2008;8(7):501-511.

8. Cao J, Yan Q. Histone ubiquitination and deubiquitination in transcription, DNA damage response, and cancer. Fron Oncol. 2012;2:26.

9. Komander D, Clague MJ, Urbe S. Breaking the chains: structure and function of the deubiquitinases. Nat Rev Mol Cell Biol. 2009;10(8):550-563.

10. Reyes-Turcu FE, Ventii KH, Wilkinson KD. Regulation and cellular roles of ubiquitin-specific deubiquitinating enzymes. Annu Rev Biochem. 2009; 78:363-397.

11. LiM, Chen D, Shiloh A, etal. Deubiquitination of 53 by HAUSP is an important pathway for p53 stabilization. Nature. 2002;416(6881):648-653.

12. Yuan J,LuoK, Zhang L, Cheville JC, LouZ. USP10 regulatesp53 localization and stability by deubiquitinating p53. Cell. 2010;140(3):384-396.

13. Brummelkamp TR, Nijman SM, Dirac AM, Bernards R. Loss of the cylindromatosis tumour suppressor inhibits apoptosis by activating NF-kappaB. Nature. 2003;424(6950):797-801.

14. Wertz IE, O'Rourke KM, Zhou H, et al. De-ubiquitination and ubiquitin ligase domains of A20 downregulate NF-kappaB signalling. Nature. 2004;430(7000):694-699.

15. Tzimas C, Michailidou G, Arsenakis M, Kieff E, Mosialos G, Hatzivassiliou EG. Human ubiquitin specific protease 31 is a deubiquitinating enzyme implicated in activation of nuclear factor-kappaB Cell Signal. 2006;18(1):83-92.

OncoTargets and Therapy

\section{Publish your work in this journal}

OncoTargets and Therapy is an international, peer-reviewed, open access journal focusing on the pathological basis of all cancers, potential targets for therapy and treatment protocols employed to improve the management of cancer patients. The journal also focuses on the impact of management programs and new therapeutic agents and protocols on
16. Schweitzer K, Bozko PM, Dubiel W, Naumann M. CSN controls NFkappaB by deubiquitinylation of IkappaBalpha. EMBO J. 2007;26(6): $1532-1541$.

17. Enesa K, Zakkar M, Chaudhury H, et al. NF-kappaB suppression by the deubiquitinating enzyme Cezanne: a novel negative feedback loop in pro-inflammatory signaling. J Biol Chem. 2008;283(11):7036-7045.

18. Xu G, Tan X, Wang H, et al. Ubiquitin-specific peptidase 21 inhibits tumor necrosis factor alpha-induced nuclear factor kappaB activation via binding to and deubiquitinating receptor-interacting protein $1 . \mathrm{J}$ Biol Chem. 2010;285(2):969-978.

19. Ye Y, Akutsu M, Reyes-Turcu F, Enchev RI, Wilkinson KD, Komander D. Polyubiquitin binding and cross-reactivity in the USP domain deubiquitinase USP21. EMBO Rep. 2011;12(4):350-357.

20. Nakagawa T, Kajitani T, Togo S, et al. Deubiquitylation of histone $\mathrm{H} 2 \mathrm{~A}$ activates transcriptional initiation via trans-histone cross-talk with H3K4 di- and trimethylation. Genes Dev. 2008;22(1):37-49.

21. Zhang J, Chen C, Hou X, et al. Identification of the E3 deubiquitinase ubiquitin-specific peptidase 21 (USP21) as a positive regulator of the transcription factor GATA3. J Biol Chem. 2013;288(13): 9373-9382.

22. Peng L, Hu Y, Chen D, Jiao S, Sun S. Ubiquitin specific peptidase 21 regulates interleukin-8 expression, stem-cell like property of human renal cell carcinoma. Oncotarget. 2016;7(27):42007-42016.

23. Yu H, Simons DL, Segall I, et al. PRC2/EED-EZH2 complex is upregulated in breast cancer lymph node metastasis compared to primary tumor and correlates with tumor proliferation in situ. PLoS One. 2012; 7(12): 51239

24. Yu MA, Kiang A, Wang-Rodriguez J, et al. Nicotine promotes acquisition of stem cell and epithelial-to-mesenchymal properties in head and neck squamous cell carcinoma. PLoS One. 2012;7(12):e51967.

25. Martinez-Fernandez M, Rubio C, Segovia C, Lopez-Calderon FF, Duenas M, Paramio JM. EZH2 in bladder cancer, a promising therapeutic target. Int J Mol Sci. 2015;16(11):27107-27132.

26. Warrick JI, Raman JD, Kaag M, et al. Enhancer of zeste homolog 2 (EZH2) expression in bladder cancer. Urol Oncol. 2016;34(6):258. e1-e6.

27. Fan Y, Mao R, Yu Y, et al. USP21 negatively regulates antiviral response by acting as a RIG-I deubiquitinase. $J$ Exp Med.2014;211(2): 313-328.

28. Heride C, Rigden DJ, Bertsoulaki E, et al. The centrosomal deubiquitylase USP21 regulates Gli1 transcriptional activity and stability. $J$ Cell Sci. 2016;129(21):4001-4013.

29. Pannu J, Belle JI, Forster M, et al. Ubiquitin specific protease 21 is dispensable for normal development, hematopoiesis and lymphocyte differentiation. PLoS One. 2015;10(2):e0117304.

30. Du Z, Tong X, Ye X. Cyclin D1 promotes cell cycle progression through enhancing NDR1/2 kinase activity independent of cyclin-dependent kinase 4. J Biol Chem. 2013;288(37):26678-26687.

31. Leontieva OV, Blagosklonny MV. CDK4/6-inhibiting drug substitutes for p21 and p16 in senescence: duration of cell cycle arrest and MTOR activity determine geroconversion. Cell Cycle. 2013;12(18): 3063-3069.

32. Bendris N, Lemmers B, Blanchard JM. Cell cycle, cytoskeleton dynamics and beyond: the many functions of cyclins and CDK inhibitors. Cell Cycle. 2015;14(12):1786-1798.

\section{Dovepress}

patient perspectives such as quality of life, adherence and satisfaction. The manuscript management system is completely online and includes a very quick and fair peer-review system, which is all easy to use. Visit http://www.dovepress.com/testimonials.php to read real quotes from published authors. 